\title{
SARS-CoV-2 Ausbruchssituation: Appell an den Bundesgesundheitsminister und die Gesundheitsbehörden der Bundesländer zur Verschiebung der Umsetzung des Vertriebsweges von Gerinnungsfaktorenzubereitungen
}

\author{
Jürgen Koscielny Günther Kappert Christoph Sucker
}

Hämostaseologie 2020;40:245.

Die aktuelle Corona-Pandemie hat erhebliche Auswirkungen auf verschiedenste Bereiche des täglichen Lebens in Deutschland. Betroffen hiervon ist in besonderer Weise auch der Gesundheitssektor, Kliniken und Praxen stehen vor nie gekannten medizinischen, logistischen und ökonomischen Herausforderungen. Anlass für den aktuellen dringenden Appell an den Bundesgesundheitsminister, ist es, auch im Rahmen der Corona-Pandemie eine adäquate Versorgung von Patienten mit hämophilen Gerinnungsstörungen zu gewährleisten.

Hintergrund für den gemeinsamen Appell, der neben dem Berufsverband der Deutschen Hämostaseologen (BDDH) auch durch die Gesellschaft für Thrombose- und Hämostaseforschung (GTH), die Deutsche Hämophilie-Gesellschaft (DHG), die Deutsche Gesellschaft für Transfusionsmedizin und Immunhämatologie (DGTI), die Interessengemeinschaft Hämophiler (IGH) sowie die Deutsche Bluthilfe unterstützt wird, ist die für den 15.08.2020 vorgesehene Umsetzung des neuen Apothekenvertriebsweges für Gerinnungsfaktorenzubereitungen für Patienten mit hämophilen Gerinnungsstörungen nach dem Gesetz für mehr Sicherheit in der Arzneimittelversorgung (GSAV). Die unterzeichnenden Patienten- und Berufsorganisationen sehen die Etablierung des neuen Vertriebsweges zum oben genannten Zeitpunkt aufgrund der aktuell durch die Pandemie sehr dynamischen Situation als problematisch an. Apotheken, Großhandel und systemrelevante ärztliche Versorgungsbereiche stehen aufgrund der Herausforderungen, die sich im Kontext der aktuellen epidemischen Entwicklung ergeben, an der Grenze der Überforderung. Die notwendigen Vorbereitungen für eine reibungslose Umsetzung des Gesetzes zum 15.08.2020 kann von dem mit dessen Ausführung betrauten Personenkreis derzeit nur zum Teil oder gar nicht umgesetzt werden. Die Änderung des Vertriebsweges während oder unmittelbar nach der Corona-Krise könnte daher die Sicherheit von Hochrisiko-Patienten mit hämophilen Gerinnungsstörungen aufgrund des ständigen Risikos für schwere Blutungen und der zum Teil bestehenden immunkompromittierenden Begleiterkrankungen hochgradig gefährden.

Die beteiligten Patienten- und Berufsorganisationen haben daher den Bundesgesundheitsminister um eine zeitliche Verlängerung des aktuell bestehenden, etablierten Vertriebsweges über die Zentrumsstruktur und teilweise auch über die Krankenhausapotheken ersucht. Hierbei ist Konsens, dass die Beibehaltung der aktuellen Strukturen des Vertriebsweges weitaus weniger Probleme für die Patientenversorgung während der Corona-Pandemie aufwerfen als eine entsprechende tiefgreifende Änderung des etablierten Vorgehens in der besonderen epidemiologischen Situation. Konkrete Forderung an das Bundesministerium für Gesundheit (BMG) und die Minister und Senatoren für Gesundheit der Bundesländer ist die Prüfung der Aussetzung der auf den 15.08.2020 terminierten Änderungen des Vertriebsweges im Rahmen des GSAV bis zur Überwindung der Corona-Krise bzw. bis zur Entwicklung einer ausreichenden Immunität in der Bevölkerung und zur Normalisierung des öffentlichen Lebens.

Es ist zu hoffen, dass dieser breit unterstützte Appell, der am 24.03.2020 an die Entscheidungsträger übermittelt wurde, berücksichtigt und entsprechend umgesetzt wird, damit trotz der derzeit aufgrund der Corona-Pandemie angespannten Situation eine reibungslose und adäquate Therapie Hämophiler gewährleistet ist.

Für den Vorstand des Berufsverbandes der Deutschen Hämostaseologen e.V. (BDDH):

Priv.-Doz. Dr. Jürgen Koscielny, Berlin, Vorsitzender

Dr. Günther Kappert, Duisburg, Stellvertretender Vorsitzender

Priv.-Doz. Dr. Christoph Sucker, Berlin, Beisitzer des Vorstands (c) 2020 Georg Thieme Verlag KG Stuttgart $\cdot$ New York
DOI https://doi.org/ 10.1055/a-1081-6879. ISSN 0720-9355. 